\title{
Sequencing a Juglans regia $\times J$. microcarpa hybrid yields high-quality genome assemblies of parental species
}

Tingting Zhu (1), Le Wang', Frank M. You², Juan C. Rodriguez', Karin R. Deal', Limin Chen', Jie Li', Sandeep Chakraborty ${ }^{1}$, Bipin Balan', Cai-Zhong Jiang (1) ${ }^{1,4}$, Patrick J. Brown ${ }^{1}$, Charles A. Leslie', Mallikarjuna K. Aradhya ${ }^{5}$, Abhaya M. Dandekar (1)', Patrick E. McGuire', Daniel Kluepfel (1)', Jan Dvorak ${ }^{1}$ and Ming-Cheng Luo (1)

\begin{abstract}
Members of the genus Juglans are monecious wind-pollinated trees in the family Juglandaceae with highly heterozygous genomes, which greatly complicates genome sequence assembly. The genomes of interspecific hybrids are usually comprised of haploid genomes of parental species. We exploited this attribute of interspecific hybrids to avoid heterozygosity and sequenced an interspecific hybrid Juglans microcarpa $\times J$. regia using a novel combination of single-molecule sequencing and optical genome mapping technologies. The resulting assemblies of both genomes were remarkably complete including chromosome termini and centromere regions. Chromosome termini consisted of arrays of telomeric repeats about $8 \mathrm{~kb}$ long and heterochromatic subtelomeric regions about $10 \mathrm{~kb}$ long. The centromeres consisted of arrays of a centromere-specific Gypsy retrotransposon and most contained genes, many of them transcribed. Juglans genomes evolved by a whole-genome-duplication dating back to the CretaceousPaleogene boundary and consist of two subgenomes, which were fractionated by numerous short gene deletions evenly distributed along the length of the chromosomes. Fractionation was shown to be asymmetric with one subgenome exhibiting greater gene loss than the other. The asymmetry of the process is ongoing and mirrors an asymmetry in gene expression between the subgenomes. Given the importance of J. microcarpa $\times J$. regia hybrids as potential walnut rootstocks, we catalogued disease resistance genes in the parental genomes and studied their chromosomal distribution. We also estimated the molecular clock rates for woody perennials and deployed them in estimating divergence times of Juglans genomes and those of other woody perennials.
\end{abstract}

\section{Introduction}

Haplotype phasing ${ }^{1}$ and navigating between allelic and nonallelic variation are the major challenges in assembling genomes of outcrossing species with high levels of heterozygosity such as found in members of the genus Juglans. Genome sequencing targeting outcrossing

Correspondence: Jan Dvorak (jdvorak@ucdavis.edu) or

Ming-Cheng Luo (mcluo@ucdavis.edu)

${ }^{1}$ Department of Plant Sciences, University of California, Davis, CA 95616, USA

${ }^{2}$ Ottawa Research and Development Centre, Agriculture and Agri-Food

Canada, Ottawa, ON K1A 0C6, Canada

Full list of author information is available at the end of the article.

These authors contributed equally: Tingting Zhu, Le Wang, Frank M. You plants employed inbred lines ${ }^{2}$, haploids ${ }^{3}$, and megagametophytes ${ }^{4}$ to avoid heterozygosity.

Interspecific hybrids offer another strategy to avoid heterozygosity. Since the genome of an interspecific hybrid is usually comprised of haploid genomes of the parental species, interspecific hybrids have the same advantages for genome sequencing as haploids, but are usually easy to produce. Technical difficulties with allocating scaffolds to parental genomes have precluded the deployment of hybrids in genome sequencing. Using an interspecific hybrid between cultivated walnut and its wild relative $J$. microcarpa, we describe here a novel approach to sequencing hybrid genomes which results in

\section{(c) The Author(s) 2019}

(c) (i) Open Access This article is licensed under a Creative Commons Attribution 4.0 International License, which permits use, sharing, adaptation, distribution and reproduction cc) in any medium or format, as long as you give appropriate credit to the original author(s) and the source, provide a link to the Creative Commons license, and indicate if changes were made. The images or other third party material in this article are included in the article's Creative Commons license, unless indicated otherwise in a credit line to the material. If material is not included in the article's Creative Commons license and your intended use is not permitted by statutory regulation or exceeds the permitted use, you will need to obtain permission directly from the copyright holder. To view a copy of this license, visit http://creativecommons.org/licenses/by/4.0/. 
a cost-effective high-quality genome assembly for both parents.

The cultivated Persian/English walnut, Juglans regia, is native to Asia whereas J. microcarpa is native to North America, where it occurs in riparian areas in the southwestern USA. Both species are wind-pollinated, highly heterozygous, and intolerant of inbreeding. Their hybrids are infertile. Both have a genome size of about $600 \mathrm{Mb}^{5}$ with $n=16$.

English walnut is an important nut crop with 3.8 million tons harvested worldwide in 2017 (http://www.fao.org/ faostat/en/\#data/QC). Walnut production has been steadily increasing in part due to health benefits derived from including walnuts in the human $\operatorname{diet}^{6-8}$. In the USA, English walnut trees are grown commercially using rootstocks chosen for their ability to tolerate such soilborne pathogens as Phytophthora spp. ${ }^{9}$, lesion nematodes, and Agrobacterium tumefaciens ${ }^{10}$, which are all serious pathogens of walnut. The commercial hybrid rootstock $J$. microcarpa $\times J$. regia possessing tolerance to soil borne diseases is extensively used in walnut production in California. The development of genomic resources for walnut and its wild relatives, including reference-quality genome sequences, will accelerate genetic improvement of walnut scions and rootstocks.

Juglans and its relatives in the family Juglandaceae are members of order Fagales, which includes many important forest trees. Reference-quality genome sequences will facilitate comparative genomics and will advance biology of this important group of woody perennials. Recent attempts to sequence the heterozygous genome of English walnut using traditional approaches resulted in assemblies with 4402 scaffolds with $\mathrm{N} 50=640 \mathrm{~kb}^{5,11}$ and 25,670 scaffolds with $\mathrm{N} 50=310 \mathrm{~kb}^{12}$. An attempt to sequence the heterozygous genome of $J$. microcarpa resulted in an even more fragmented assembly with 329,873 scaffolds with $\mathrm{N} 50=136 \mathrm{~kb}^{5}$. The novel sequencing approach described here exploits the synergy between long-read sequencing and optical genome mapping. The average length of reads produced with longread platforms exceeds the lengths of a vast majority of plant long-terminal-repeat retrotransposons (LTR-RTs), which results in a dramatically improved sequence assembly ${ }^{13}$. Contigs or scaffolds assembled from longreads are sufficiently long to be aligned on genome-wide optical maps, which can be assembled with very high accuracy, even for large or polyploid plant genomes ${ }^{14,15}$. Alignments of the optical maps of a hybrid onto the optical maps of its parents will assign contigs to parental genomes ${ }^{16}$, while also serving as an assembly quality control $^{14}$.

We used the assembled J. regia and J. microcarpa genome sequences in conjunction with the Juglandoid wholegenome-duplication (WGD) ${ }^{17}$, and the Juglandaceae fossil record to calibrate the molecular clock rate for woody perennials. We used the calibrated molecular clock to estimate the time of divergence of Juglans species and other woody perennials. Based on synteny within Juglans genomes, we allocated the 16 Juglans chromosomes produced by the Juglandoid WGD into eight homoeologous chromosome pairs and analyzed their evolution. Finally, we exploited the contiguity of the assemblies in the analyses of the structure and evolution of Juglans telomeres and centromeres and the distribution of disease resistance genes in the J. regia and J. microcarpa genomes.

\section{Results}

\section{Genome assembly}

We sequenced 58 Pacific Biosciences SMRT cells of the hybrid (MS1-56) J. microcarpa 31.01 (henceforth 31.01) $\times$ J. regia cv Serr (henceforth Serr) and assembled the reads (Table S1 and Fig. S1) into 460 polished contigs (for a flowchart of the entire assembly see Fig. S2). The total length of this assembly (MS1-56_v0) was 1,056,053,408 bp (N50 = 7,963,037 bp) (Table S2).

Next, we constructed two optical maps for the hybrid and one for each of its parents (Table S3). The N50 of the optical contigs ranged from 1.31 to $2.90 \mathrm{Mb}$. The parental maps consisted of 'haploid' regions, in which the haplotypes were similar enough to collapse into a single contig, and 'diploid' regions, in which the haplotypes were dissimilar enough to be assembled into separate contigs (phased). The haploid and diploid regions were identified by a map self-alignment (Fig. S3). The mean length across the phased regions was $108 \mathrm{Mb}(16.8 \%)$ in Serr and $33 \mathrm{Mb}$ $(5.9 \%)$ in 31.01 (Table S4). Due to the inclusion of phased haplotypes in a map, the sum of the total lengths of the Serr and 31.01 optical maps was $13.4 \%$ longer than the length of the MS1-56 optical map (Fig. S3).

To ascertain whether the genome of our hybrid was complete, we compared the total length of its optical map to the sum of the lengths of the optical maps of the parents, which we edited to disregard the redundant haplotypes from the phased regions (Fig. S3). The total length of the edited maps of Serr and 31.01 differed from the length of the map of MS1-56 by only $1 \mathrm{Mb}(0.09 \%)$ (Table S4). We therefore concluded that the hybrid genome was a complete representation of the parental genomes.

We aligned the sequence contigs on the optical map of the hybrid, stitched them into scaffolds (assembly MS156_scf, total length $=1,066,408,726 \mathrm{bp}, \mathrm{N} 50=34,776,948$ bp), and allocated 40 of them comprising $99.85 \%$ of the hybrid genome assembly into the parental genomes with the aid of the optical maps of the parents (Fig. S3). The remaining 224 scaffolds representing $0.15 \%$ of the hybrid genome sequence were too short $($ mean $=7.2 \mathrm{~kb})$ to be aligned on an optical map and were aligned on Illumina 
sequence of the $J$. regia $\mathrm{cv}$ Chandler ${ }^{11}$ for assigning to parental genomes.

Finally, we ordered and oriented scaffolds on highdensity genetic maps producing 16 pseudomolecules for each of the two genomes. They had only five gaps of unknown lengths (Fig. S4); the remaining gaps were estimated based on the optical map alignments. We then reduced gap lengths or closed them entirely with unassigned contigs or contigs produced with the 10X Genomics technology (Table S1).

We mapped 89X Illumina reads of the hybrid to the genome assemblies and detected 48,717 and 51,789 indels and 748 and 1,205 base substitutions in the Serr and 31.01 assemblies, respectively (about one error per 10,000 bp). We subsequently corrected these errors and produced the final assemblies JrSerr_v1.0 and Jm31.01_v1.0 (Table S5).

\section{Transposable element and gene annotation}

Transposable elements (TEs) represented 41.02 and $40.86 \%$ of the JrSerr_v1.0 and Jm31.01_v1.0 assemblies, respectively (Table S6). The density of the Copia LTRRTs decreased and that of the Gypsy LTR-RTs increased from the chromosome ends towards the centromeres (Fig. 1a and S5). The JrSerr_v1.0 and Jm31.01_v1.0 assemblies each contained $0.03 \%$ satellite DNA and 351,359 and 349,951 simple sequence repeats (SSRs), respectively (Table S7). SSR density was highest in the distal regions of the pseudomolecules (Fig. S6).

We annotated 31,425 and 29,496 high-confidence (HC) protein-coding genes in the JrSerr_v1.0 and Jm31.01_v1.0 assemblies; 31,286 and 29,397 were on the pseudomolecules and 139 and 99 were on unassigned scaffolds (Table S8, S9). Of 1,440 BUSCO v3 genes, 96.0 and $95.2 \%$ were among the $\mathrm{HC}$ genes annotated in the JrSerr_v1.0 and Jm31.01_v1.0 assemblies, which is comparable to coverage reported in other genome annotations in plants (Table S10). Serr and 31.01 genes were on average 4,226 and 4,391 bp long and had 5.44 and 5.60 exons averaging 258.64 and 249.38 bp (Table S8). Transcription factors were encoded by 2,046 (6.5\%) and 2,032 (6.9\%) genes in the JrSerr_v1.0 and Jm31.01_v1.0 assemblies (Table S11). There were 18,772 (59.7\%) and 18,891 (64.0\%) single-copy genes and 744 and 625 multigene families in the JrSerr_v1.0 and Jm31.01_v1.0 assemblies. The larger number of genes annotated in the J. regia genome compared to the J. microcarpa genome was due to the larger number of duplicated genes in the former.

Average gene density was one gene per 16.9 and $17.7 \mathrm{~kb}$ in the JrSerr_v1.0 and Jm31.01_v1.0 pseudomolecules. Gene density decreased and TE density increased from pseudomolecule ends to centromeric regions (Fig. 1a and S5), and the two variables were negatively correlated
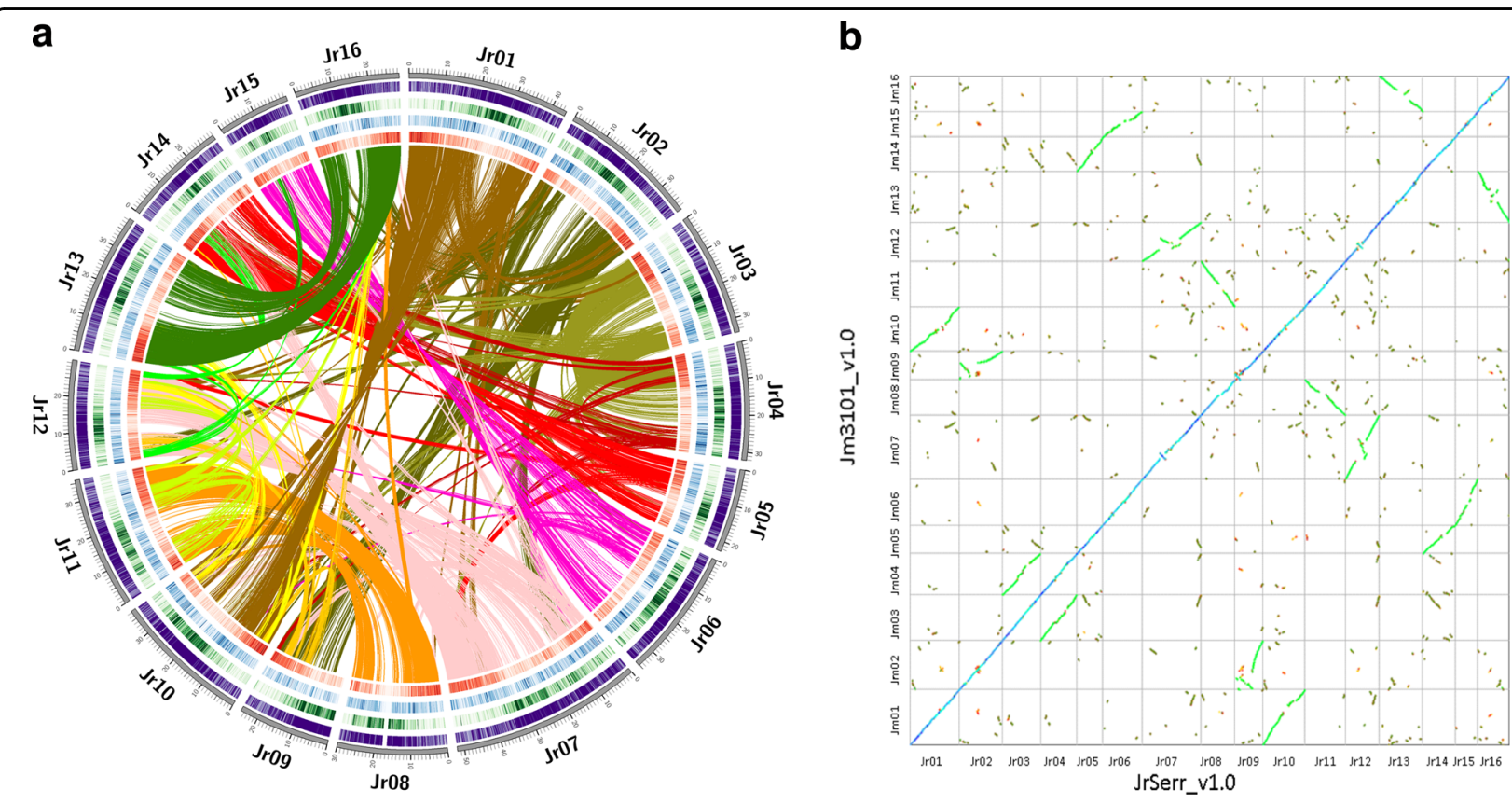

Fig. 1 Structure and phylogeny of Juglans genomes. a Circular plot of the Juglans regia pseudomolecules. Circles from outside to inside are: pseudomolecule coordinates in Mb, density of all types of LTR-RTs, density of Gypsy LTR-RTs, density of Copia LTR-RTs, and gene density. Central lines connect syntenic blocks across chromosomes. Different colors represent different homoeologous chromosome pairs derived from the Juglandoid WGD. $\mathbf{b}$ Dot-plot alignments of the JrSerr_v1.0 pseudomolecules against Jm31.01_v1.0 pseudomolecules. The starts of the pseudomolecules are to the left or bottom. The blue diagonal across the plots of the 16 chromosomes indicate collinearity of homoeologous chromosomes between the two genomes. The green dots indicate homoeologous chromosomes within a genome, which originated by the Juglandoid WGD. The short black and red dot traces indicate paralogues produced the $\gamma$ whole genome triplication 
$(r=-0.82, p<0.01)$. Gene density was positively correlated with meiotic recombination rates ${ }^{17}$ along the J. regia pseudomolecules $(r=0.78, p<0.01)$.

\section{Juglans genome structure and evolution}

Of the genes annotated on the Serr pseudomolecules, $26,403(84.4 \%)$ were collinear with genes on the J. microcarpa pseudomolecules (File S1). The two genomes differed by 28 inversions involving $>3$ collinear genes, 21 segmental duplications, 3 intra- and 14 inter-chromosomal interstitial translocations, but no terminal translocation (File S1, Fig. 1b). Only $90(0.28 \%)$ of the JrSerr_v1.0 genes were not detected in the Jm31.01_v1.0 assembly.
We computed $K s$ divergence among the J. regia and J. microcarpa genes to analyze gene duplication and divergence. The Ks plot showed three peaks (Fig. 2a). The first peak mostly consisted of $K s$ values between orthologous genes in the two genomes and reflected their divergence. The second peak coincided with the major $K s$ peak in self-searches within the Serr and 31.01 genomes and reflected the divergence of paralogous genes, which originated by the Juglandoid WGD. The third peak coincided with the major peak in self-searches within the grape (Vitis vinifera) genome and reflected divergence between genes duplicated by the whole genome triplication $\left(\gamma\right.$ WGT) first described in the grape genome ${ }^{18}$. These
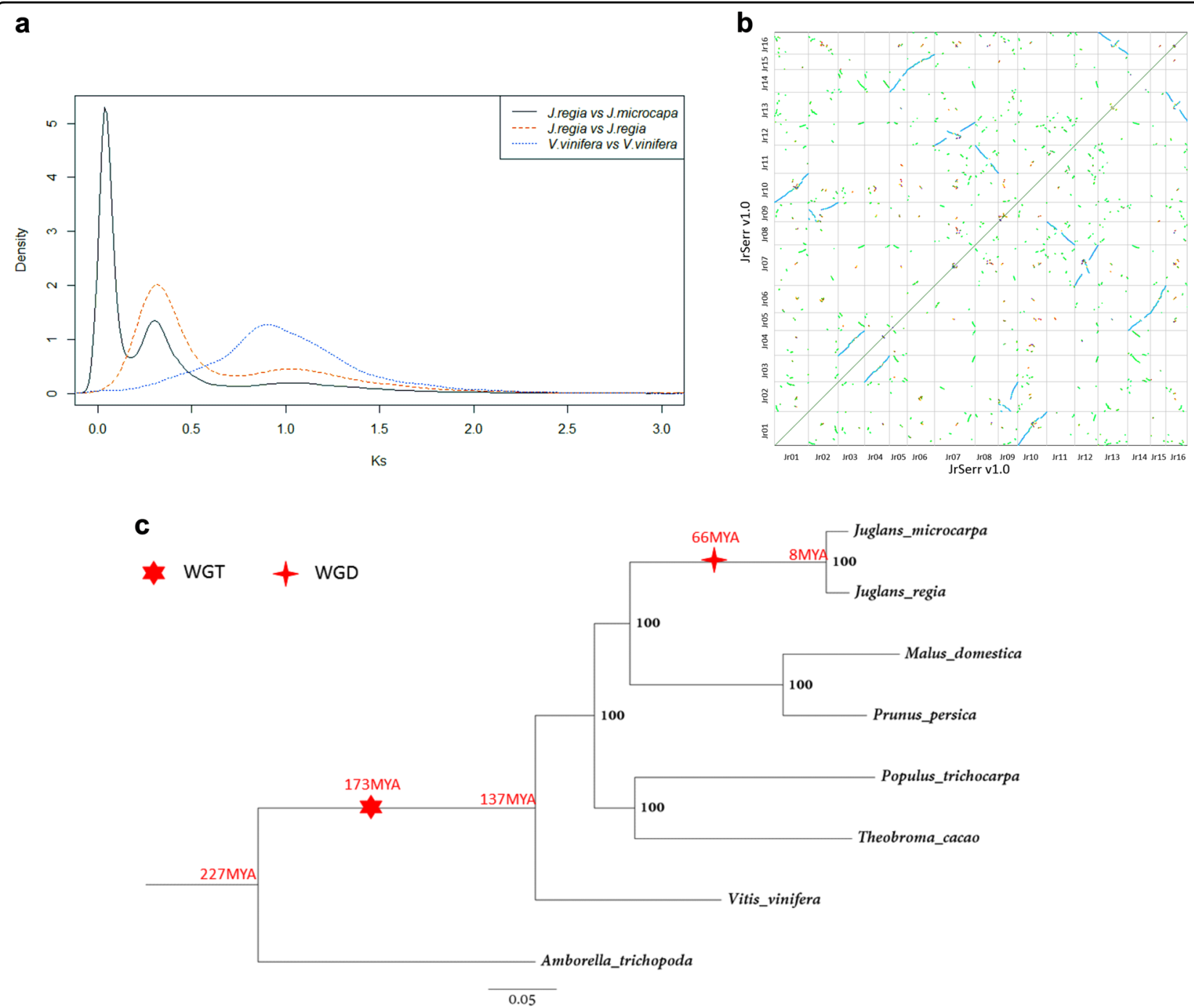

Fig. 2 Whole-genome duplication and triplication in the Juglans lineage. a Densities of Ks divergence in the BLASTP homology searches of JrSerr_v1.0 against Jm31.01_v1.0, within JrSerr_v1.0, and within the grape genome ${ }^{18}$. b Self-alignments of the J. regia Serr pseudomolecules. Pseudomolecules are oriented as in 1b. c Divergence of the genomes of Vitis vinifera, Prunus persica, Amborella trichopoda, Populus trichocarpa, Theobroma cacao, Malus domestica, J. regia cv Serr, and J. microcarpa acc. 31.01. The dendogram was constructed from Ka divergence of 1155 singlecopy genes. Bootstrap confidence on each node is shown. Also shown are estimated times of critical events in Juglans genome evolution, including the YWGT and the Juglandoid WGD (Table S13) 
inferences were confirmed by self-alignment plots (Figs. 1a, 2b, S5, File S1), a plot of the JrSerr_v1.0 pseudomolecules against the grape pseudomolecules (Fig. S7), and a plot against the Amborella trichopoda scaffolds ${ }^{19}$ (Fig. S8).

With the sole exception of Rhoiptelea chiliantha, all species of Juglandaceae share $n=16$ or a chromosome number derived from $\mathrm{it}^{20}$. Rhoiptelea chiliantha and the neighboring family Myricaceae have $n=8 ; n=16$ was derived from $n=8$ by the Juglandoid WGD ${ }^{17}$. The fossil record showed that Juglandaceae appeared in the upper Cretaceous and the family radiated in the Paleocene ${ }^{21-23}$. The Juglandoid WGD must have therefore originated prior to the radiation of Juglandaceae in the Paleocene but after the divergence of $R$. chiliantha in the Cretaceous. We therefore chose the Cretaceous-Paleogene (C-Pg) boundary (66 MYA) as the approximate time of the origin of the Juglandoid WGD. Of the 31,286 genes on the JrSerr_v1.0 pseudomolecules, 12,195 (39.0\%) were genes duplicated by the Juglandoid WGD that were in collinear locations on the homoeologous chromosomes. Using the WGD time estimate and the average $K a$ and $K s$ between these paralogues in the Serr and 31.01 genomes, we estimated clock rates as $5.6 \times 10^{-10}$ nucleotide substitutions year ${ }^{-1}$ using $K a$ and $2.5 \times 10^{-9}$ nucleotide substitutions year ${ }^{-1}$ using Ks (Table S12).

To date the divergence of J. regia and J. microcarpa, we identified 1155 single-copy genes shared by J. regia, J. microcarpa, and six other sequenced genomes of woody perennials, including grape and A. trichopoda, and constructed a dendogram (Fig. 2c). Its topology was consistent with branching of the tree of life (http://tolweb. org/Core_Eudicots/20714). However, the dating of J. regia and $J$. microcarpa divergence, about 8 MYA, was one-half to one-quarter of previously reported estimates ${ }^{5,24,25}$. Our estimates of divergence time of the Amborella lineage, the grape lineage, and the origin of the $\gamma$ WGT (Table S13) would be more recent than the published data or fossil records ${ }^{26-28}$ if this discrepancy were caused by an incorrectly calibrated molecular clock or incorrectly computed divergence times. However, this was not the case.

If we failed to correctly phase the J. regia and J. microcarpa haplotypes during the assembly of the hybrid genome sequence, orthologous genes in our JrSerr_v1.0 and Jm31.01_v1.0 assemblies would appear more closely related to each other than they actually are and the age of divergence of the two genomes could be underestimated. To test this possibility, we computed the $K a$ and $K s$ values and time of divergence between J. regia and J. microcarpa using sequences of 1077 single-copy genes independently sequenced by Stevens et al. ${ }^{5}$. The $K a$ and $K s$ values we obtained were similar to those computed from our gene sequences (Table S13). We then constructed a chronogram including genomes of peach, apple, poplar, and cocoa and the six Juglans genomes reported by Stevens et $\mathrm{al}^{5}$. in addition to the genomes used in Fig. 2c (Fig. S9). The topology of the chronogram was similar to that in Fig. $2 \mathrm{c}$ and the divergence time estimates of the branches shared with Fig. 2c were within the 95\% confidence intervals of estimates in Fig. 2c (Table S13). Based on these analyses, we suggest that the discrepancies in dating of the J. regia and J. microcarpa divergence is due to the use of an unrealistic rate of the molecular clock in the previous studies.

We analyzed gene collinearity along homoeologous chromosomes within the Serr genome and detected 38 paracentric inversions involving $>3$ collinear genes and 20 intra- and 16 inter-chromosomal interstitial translocations. The homoeologues did not differ by any terminal translocations and retained a 1:1 relationship. Using the grape genome as an outgroup, we assigned rearrangements to phylogenetic branches (File S1) and computed the rates of their accumulation. The rates ranged from 0.4 to 1.4 major rearrangements per MY (Table S14).

The collinearity analysis showed that the $16 \mathrm{~J}$. regia chromosomes can be built from 142 major synteny blocks making up the 19 grape chromosomes. Of them, 122 $(85.9 \%)$ were shared by the J. regia homoeologous chromosomes, and were in the same order along the them (File S1 and Table S15). These rearrangements must have taken place prior to the Juglandoid WGD. We found $43.7 \%$ of the $J$. regia genes collinear with genes on the grape pseudomolecules (Table S16).

\section{Asymmetric (biased) fractionation of the Juglandoid WGD}

In each pair of homoeologous chromosomes within a Juglans genome, one chromosome contained more genes than the other (Fig. 3a). We denoted the homoeologues with more genes as "dominant" and those with fewer genes as "subdominant," allocated the 16 Juglans chromosomes into eight homoeologous chromosome pairs, which we arranged in descending order based on the number of genes in the dominant chromosome (Fig. 3a), and renamed the chromosomes (Table S17).

There were 18,179 and 17,093 genes in the dominant subgenome and 13,107 and 12,304 genes in the subdominant subgenome in JrSerr_v1.0 and Jm31.01_v1.0, respectively (Table S18). Both dominant and subdominant subgenomes had fewer genes than are in the A. trichopoda genome $\left(26,846\right.$ genes) ${ }^{19}$, which was not subject to a recent WGD (Fig. 2c). This comparison indicates that both dominant and subdominant Juglans subgenomes have lost genes since the WGD.

The difference in gene loss between dominant and subdominant homoeologues was nearly constant among the homoeologous pairs (Fig. 3a), suggesting that the rate of gene loss was intrinsic to a subgenome. The rate of gene loss was also nearly constant along each 

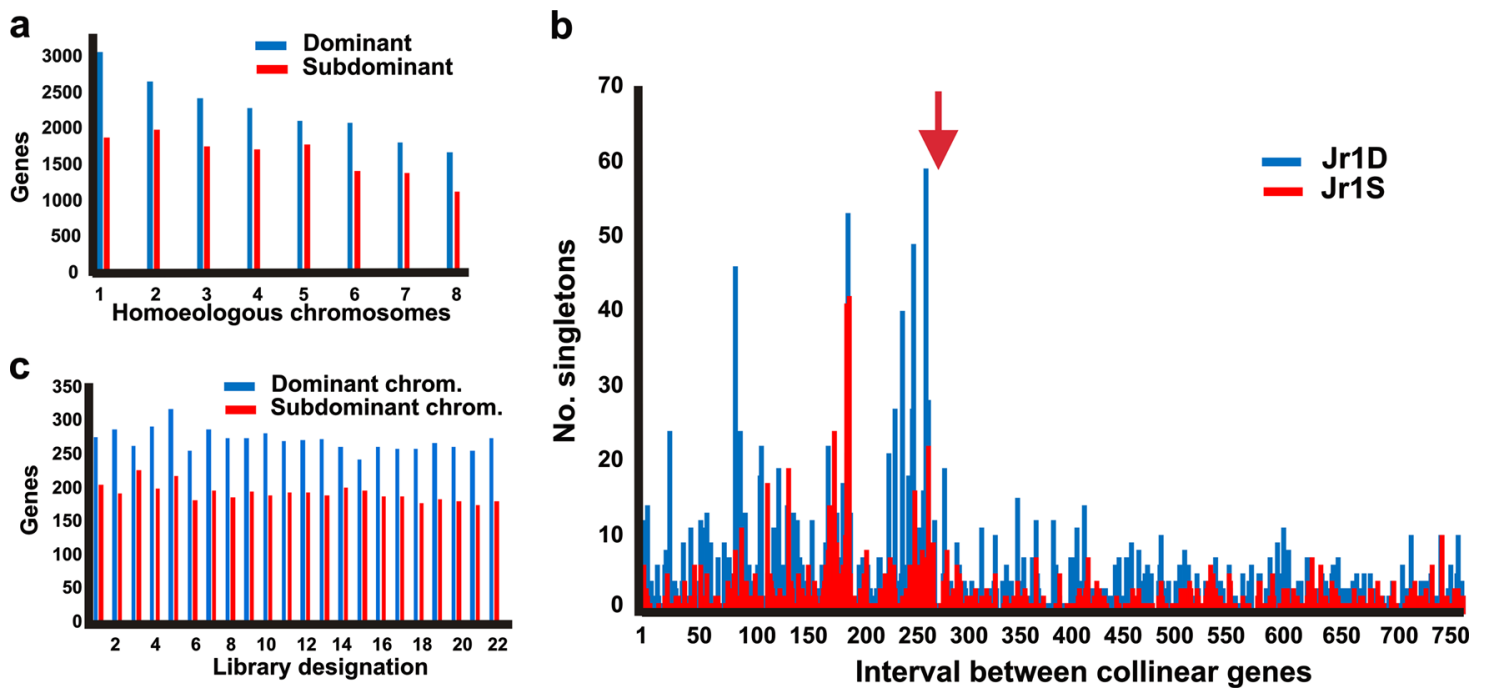

Fig. 3 Asymmetric gene fractionation of the Juglandoid WGD. a Numbers of genes annotated on the JrSerr_v1.0 dominant and subdominant pseudomolecules. Numbers on the horizontal axis are designations of individual Juglans homoeologous groups, each consisting of one dominant (D) and one subdominant (S) chromosome. $\mathbf{b}$ Numbers of singleton genes in intervals delimited by conserved paralogues on the dominant chromosome Jr1D and subdominant homoeologues Jr1S. The homoeologous pseudomolecules Jr1D and Jr1S were subdivided into intervals delimited by successive pairs of paralogous genes in collinear locations on the homoeologues. The noncollinear genes (singletons) were counted in each interval (histogram bars) in the dominant pseudomolecule (blue) and subdominant pseudomolecule (red). The starting nucleotides of the pseudomolecules are to the left. The red arrow indicates the location of the centromere in the dominant pseudomolecules. c Numbers of genes on the pseudomolecule of the dominant chromosome Jr1D (blue) and subdominant chromosome JriS (red) showing dominant expression (two-fold higher expression than that of the orthologous gene) in 22 different Juglans regia RNAseq datasets. The description of the RNAseq datasets (coded at the horizontal axis of the graph) is in Fig. S12 and Table S20

chromosome. We subdivided homoeologous chromosomes into sections delineated by successive pairs of collinear genes and counted the numbers of singleton genes in the intervening intervals (Table S19). In the JrSerr_v1.0 pseudomolecules, 13,791 singletons were on the dominant chromosomes (mean of 3.2 per interval) but only 8,261 were on the subdominant chromosomes (mean of 1.9 per interval, $p=0.0001$, paired $t$-test, $N=16$ ). The numbers of singletons per interval varied little along each chromosome, except for the proximal regions, indicating that gene loss was uniform along the chromosomes and occurred by many deletions involving one or few genes along a chromosome (Fig. $3 \mathrm{~b}$ and S10). In each chromosome, deletions were larger and more frequent in the proximal region than the rest of the chromosome, as the numbers of singletons per interval were greatly elevated in proximal regions. We also expressed the numbers of singletons per $2-\mathrm{Mb}$ non-overlapping windows. Most of the windows in subdominant chromosomes contained fewer singletons than those in the dominant homoeologues (Fig. S11).

Since the divergence of the J. regia and J. microcarpa lineages 8 MYA, $28.7 \%$ of singletons were lost from the subdominant chromosomes in J. microcarpa but only $14.9 \%$ from the corresponding intervals in the dominant homoeologues ( $p=0.002$, two-tailed paired $t$-test). Thus, the factor that caused the asymmetry in fractionation has persisted in the subgenomes since their origin to the recent past. In 22 RNAseq datasets (Table S20), genes on the dominant chromosomes were on average transcribed significantly more than their paralogues on the subdominant chromosomes (paired $t$-test, $p<1.0 \times 10^{-8}$ ) (Fig. 3c and S12, File S2).

\section{rRNA genes}

We failed to assemble the sequences of the arrays of $18 \mathrm{~S}$ 5.8S-26S rRNA genes, although we were able to assemble an optical map of the locus on Jr4S (Fig. S13). The locus was $1,310,076 \mathrm{bp}$ long $(16,646,858-17,956,934 \mathrm{bp})$ and contained 112 gene units repeated in tandem; only on unit was present in the pseudomolecule.

\section{Telomeres}

Complete arrays of the telomeric repeat CCCTAAA were present at $16 \mathrm{~J}$. regia and $11 \mathrm{~J}$. microcarpa termini (Table S21). These termini had a tripartite structure (Fig. 4). The telomeric regions consisted of monotonous arrays of telomeric repeats, with a sole exception of Jm6S which included a 47-bp (T) $)_{n}$ SSR (File S3). The subtelomeric region spanned the distance from the last telomeric repeat to the first gene and had the characteristics of heterochromatin by consisting of various microsatellites interspersed with TEs. Juxtaposed to the subtelomeric region was the euchromatic region 


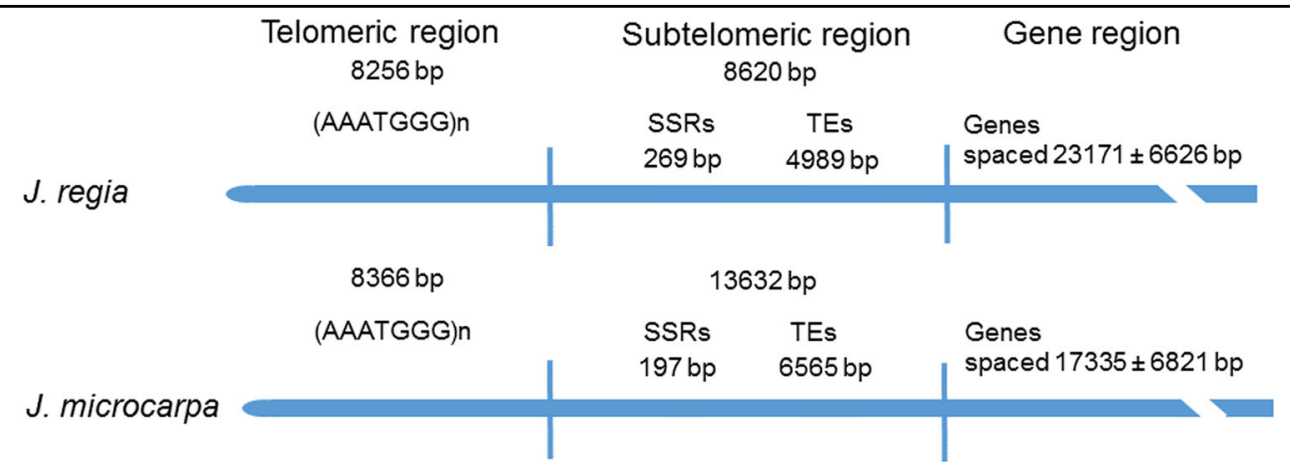

Fig. 4 Structure of chromosome termini in the Juglans regia and J. microcarpa pseudomolecules. The telomeric region consists entirely of telomeric repeats. The subtelomeric region consists of satellite motifs and TEs. The gene region starts with the coding sequence of the first gene. Mean intergenic distances and 95\% confidence intervals ( $N=16$ and 11 for J. regia and J. microcarpa, respectively) were computed for the first three protein-coding genes

containing genes. Spacing of genes was estimated for the three most distal genes and was similar to the genome average (Fig. 4).

We anticipated finding a transitional zone containing degraded telomeric repeats near the boundaries between the telomeric and subtelomeric regions. However, the boundaries were almost always sharp; the last perfect telomeric repeat was juxtaposed to unrelated sequences in the subtelomeric region (File S4). This pattern would emerge if the entire telomeric region was occasionally forged by the telomerase de novo in the germline.

\section{Centromeres}

There was one specific region greatly enriched for repeats and impoverished for genes in each J. regia (Fig. S14) and J. microcarpa pseudomolecule. These regions were at similar locations on the corresponding J. regia and J. microcarpa pseudomolecules, contained the same sequences, and had similar structure (Fig. S15). They coincided with the locations of the J. regia centromeres ${ }^{17}$. To further characterize these regions, we generated selfsynteny dot-plots for a 5 - Mb region surrounding each repeat peak. These analyses revealed the existence of clusters of a 12.5-kb Gypsy LTR-RT (Fig. 5a), which we name JCR (Juglandoid Centromeric Repeat) (Fig. 5b). JCRs were nearly exclusively located in the centromeres. For instance, we found 62 complete JCRs within a $3-\mathrm{Mb}$ centromeric region of Jr2D (Fig. 5a), but only four copies in the remaining $41 \mathrm{Mb}$ of that pseudomolecule. Analogous CRs of the Ty/Gypsy superfamily have been located in the centromere cores in grasses ${ }^{29,30}$ cotton $^{31}$, soybean $^{32}$, beet ${ }^{33}$, and Brassica ${ }^{34}$.

We located 403 protein-coding genes, many conserved and showing collinearity with genes in the grape genome, within the JCR arrays (File S1). For 201 of them, we found transcripts in two or more of the 22 RNAseq datasets we analyzed, indicating that many of these genes are expressed (File S5).
We used genes located within and near the JCR arrays and collinear on Serr dominant and subdominant homoeologous pseudomolecules as landmarks for comparisons of the locations of homoeologous centromeres within the Serr pseudomolecules (Figs. 5c, d). In the homoeologous chromosome pairs Jr4D-Jr4S, Jr5D-Jr5S, and Jr8D-Jr8S (Fig. 5c), the JCR array was located in corresponding intervals (Fig. S16). In the remaining pairs, the $J C R$ array was located in differing locations. In the Jr2D-Jr2S pair the array was repositioned to a new location without perturbing the order of the collinear genes (Fig. 5d). In that pair, centromere repositioning likely occurred by an intrachromosomal translocation of the $J C R$ array into a new location. In homoeologous chromosome pairs Jr1D-Jr1S, Jr6D-Jr6S, and Jr7D-Jr7S, the $J C R$ array was repositioned to a new location by an inversion with one breakpoint within the $J C R$ array (Fig. S16). If a break were at the edge of the JCR array, such an inversion may reposition the entire $J C R$ block and the entire centromere. If the break were in the middle of the array, it would create pair of $J C R$ arrays, as seen in Jr1S (Fig. S16). In this specific case, it needs to be determined which of the $J C R$ arrays is the active centromere and which is silent.

We frequently found inversions to terminate in the centromeric regions, suggesting that centromeric regions were prone to rearrangements (File S1). The centromeric regions were also prone to gene transposition as shown by the comparison of gene collinearity between the JrSerr_v1.0 and Jm31.01_v1.0 pseudomolecules (Fig. S17).

\section{Disease resistance genes}

Since J. microcarpa is important for the development of disease-resistant rootstocks for walnut commercial production, we paid special attention to resistance gene analogues (RGA, henceforth $\mathrm{R}$ genes) in the two genomes (File S6). There was very little difference in the number of 


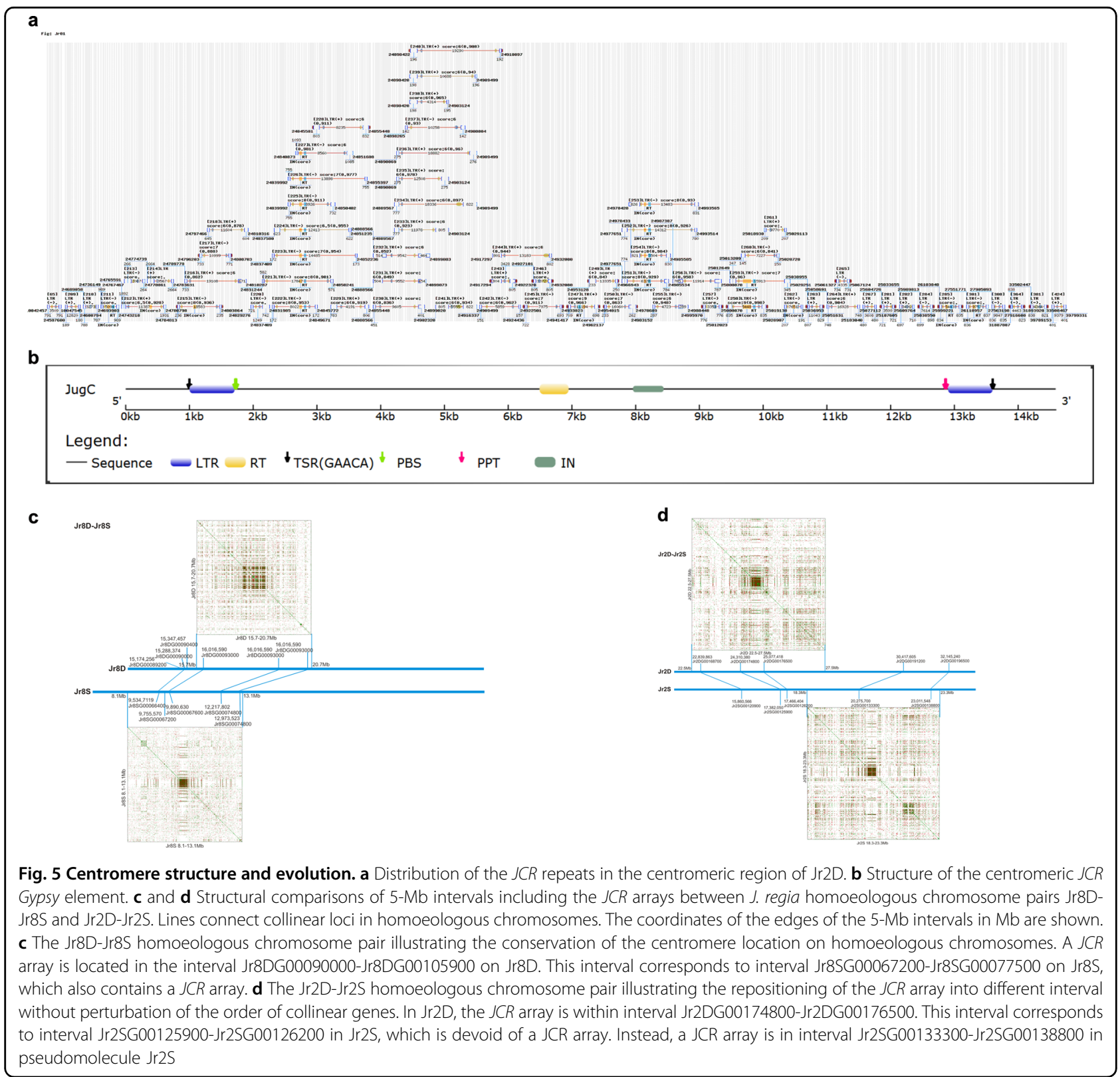

$\mathrm{R}$ genes between a cultivated and a wild species. There were $942 \mathrm{R}$ genes in JrSerr_v1.0 and 903 in Jm31.01_v1.0. The $\mathrm{R}$ genes represented $3 \%$ of the total number of genes in both genomes.

We classified the R genes into 11 categories (Table S22). The most frequent category was the receptor-like kinase (RLK), which represented 62 and $58 \%$ of the R genes in JrSerr_v1.0 and Jm31.01_v1.0 assemblies.

$\mathrm{R}$ genes were present on all chromosomes, but showed a tendency to be located in the distal regions (Fig. 6). We divided the total number of genes present in a chromosome arm into 20 windows containing equal numbers of genes and computed the proportion of $\mathrm{R}$ genes in each window. The proportions correlated with bin locations on the telomere-to-centromere axis in the JrSerr_v1.0 pseudomolecules $(r=-0.48, p=0.033)$ but in the Jm31.01_v1.0 pseudomolecules the correlation was not significant $(r=-0.35, p=0.095)$. The distribution of $\mathrm{R}$ gene was similar between homoeologous $J$. regia and $J$. microcarpa pseudomolecules, but, there was no similarity in their distribution between homoeologous chromosomes within a genome (Fig. 6).

Of the $942 \mathrm{R}$ genes in the JrSerr_v1.0 assembly, 793 (84.2\%) were in collinear location in the Jm31.01_v1.0 assembly. This was nearly identical to the $84.4 \%$ collinearity for the remaining 30,345 genes in the Serr genome. A total of $378(40.1 \%)$ of the $\mathrm{R}$ genes were in collinear locations on the Serr homoeologous 

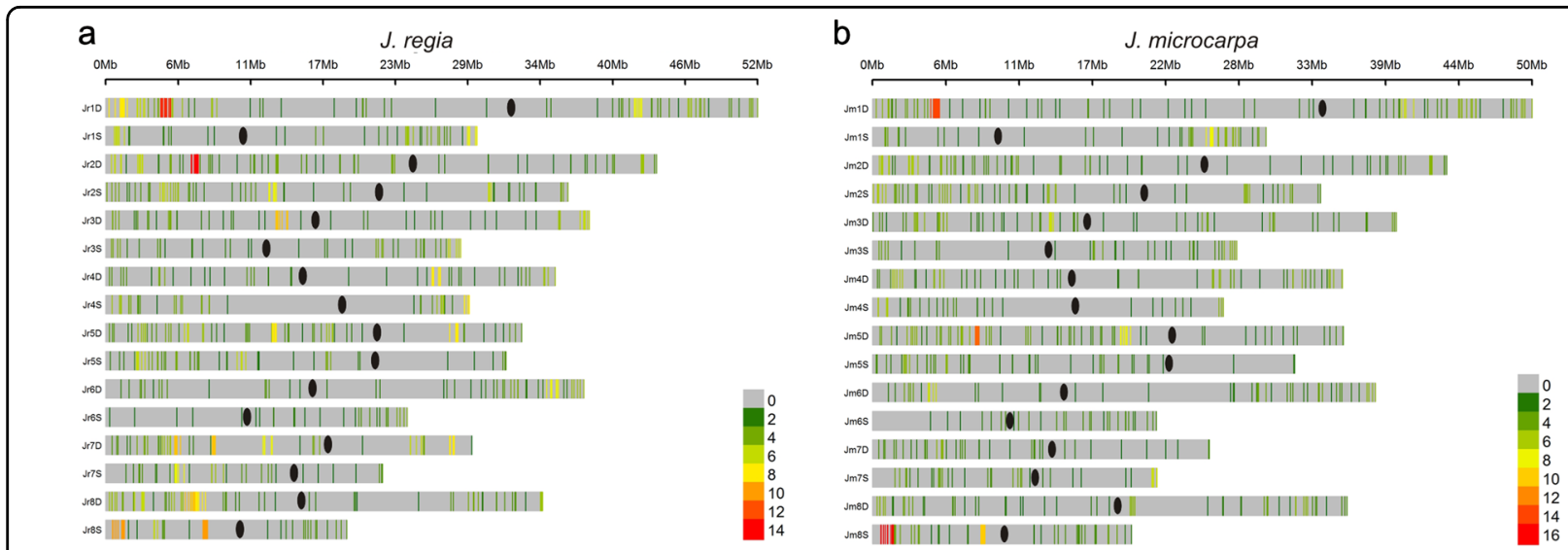

Fig. 6 The distribution of the R genes along J. regia Serr (a) and J. microcarpa 31.01 (b) chromosomes. Heat maps show the numbers of R genes per $1 \mathrm{Mb}$ non-overlapping windows along the pseudomolecules. The starting nucleotides of the pseudomolecules are to the left. Centromeres are indicated by black ovals

chromosomes. Of the remaining Serr genes, 39.0\% were in collinear locations on the homoeologous chromosomes. Therefore, there appears to be no difference in dynamism of the R gene families and the rest of the Juglans genes.

\section{Discussion}

\section{Sequencing approach}

Sequencing of heterozygous Juglans genomes using the short-read technology resulted in assemblies with short contigs and scaffolds ${ }^{5,11,12}$. Similar results were previously obtained for other outcrossing woody perennials with the Sanger sequencing technology ${ }^{18,35-37}$, which is often considered as the gold standard of DNA sequencing. We attribute the great increase in the contiguity of our assemblies compared to the previous attempts to sequence Juglans genomes to the use of a long-read sequencing technology ${ }^{38}$ in addition to the haploid state of the J. regia and J. microcarpa genomes in the interspecific hybrid.

A well-known problem accompanying the long-read sequencing technologies is high error rates. These errors must be corrected during genome assembly. We were able to correct most of the errors during polishing of the assemblies and achieved a rate of one error in 10,000 nucleotides, which matched a standard recommended for the human genome for the Sanger sequencing technology $^{39}$. In both genomes, $98 \%$ of errors that remained in the assembly after polishing were indels, indicating that the polishing algorithm was more efficient in detecting base substitution errors than base indel errors. We corrected both types of errors by mapping Illumina reads into the polished assemblies.

Long contigs obtained with the PacBio long-read technology for the J. microcarpa $\times J$. regia hybrid were critical for efficient aligning sequence contigs on the optical maps of the parental species and their allocation to parental genomes. We were able to allocate $99.85 \%$ of the hybrid genome sequence to the parental genomes. Contigs that are too short to be aligned on optical maps can be allocated to parental genomes by aligning them on Illumina reads of the parental genomes. In our case, we used the published Illumina sequence of $J$. regia cv Chandler ${ }^{11}$ for allocating short contigs to parental genomes.

The optical maps played additional roles in our genome sequence assembly. They facilitated construction of long scaffolds, which reached $\mathrm{N} 50=34.8 \mathrm{Mb}$ and approached or equaled the lengths of the chromosomes. They also facilitated detection of misassembled contigs and the estimation of the actual lengths of most of the gaps. By using two independently made optical maps for each genome (one for the hybrid and one for each parent) we were able to decide whether a discrepancy between an optical map and a sequence was due to an error in the sequence or in the map.

A synergy between long-read sequencing technology and optical mapping made it possible to leverage the haploid state of the genomes in the J. microcarpa $\times$ J. regia hybrid to produce remarkably complete genome assemblies. A concern about using a hybrid for the construction of a reference genome sequence is that in some instances one of the parental genomes is partially or completely eliminated from a hybrid. The Hordeum vulgare $\times H$. bulbosum hybrids are the best known example ${ }^{40}$. Loss of $10 \%$ or more of DNA from a genome in artificial polyploids produced by the colchicine treatment of interspecific hybrids has been reported and may be another reason for concern ${ }^{41-44}$. To ascertain whether the genome of our hybrid was complete, we compared the total length of its optical map to the sum of the lengths of the optical maps of the parents. The maps differed by a mere $0.09 \%$, which is below the error rate of these measurements. We therefore concluded that the hybrid genome was a 
complete representation of the parental genomes. The main source of genetic instability in hybrids and polyploids is meiosis ${ }^{45}$. Since DNA used for the construction of sequencing libraries and optical maps is isolated from young somatic tissues this cause of instability is irrelevant. Nevertheless, the possibility of somatic instability of an interspecific hybrid should be kept in mind and the additivity of the hybrid genomes should be checked. Making the use of the optical map trio, as we did, is the most practical approach, because it requires very little additional work.

\section{Juglans genome evolution}

We showed that Juglans genomes consist of eight pairs of homoeologous chromosomes, which originated by the Juglandoid WGD. The chromosomes were subjected to a highly asymmetric fractionation. Similar asymmetric fractionation following WGD was described in other species ${ }^{46-49}$. In wheat, the larger B genome has lost twice as much DNA as the smaller A genome since the origin of tetraploid wheat about $0.3 \mathrm{MYA}^{47}$. If a difference in subgenome sizes were the cause of asymmetric gene loss in walnut, the subgenomes should differ in gene density, since large genomes are less gene dense than small genomes. That was not the case. Gene density was similar in the dominant and subdominant homoeologues.

We showed that the asymmetric fractionation of Juglans subgenomes has not relented in the recent past. The factor that caused the asymmetry is therefore likely active in the current Juglans genomes. We suggest that the factor is asymmetric gene expression on dominant and subdominant chromosomes. An asymmetry in expression of genes on dominant and subdominant homoeologues has been reported in plant families as diverse as Poaceae $^{50,51}$, Brassicaceae $^{48}$, and Malvaceae ${ }^{49}$. A relevant question is what causes the asymmetry in gene expression. In Arabidopsis and Brassica, reduced gene expression was linked to the silencing effects of small, 24-nt RNAs amplified against specific LTR-RTs ${ }^{52}$. The methylation of LTR-RTs, and their contribution to the pool of siRNAs in walnut should be analyzed to determine if the same mechanism is the basis for the differences in gene expression in the Juglans subgenomes.

The rates of accumulation of structural changes in the Juglans genomes ranged from 0.4 to 1.4 major rearrangements per MY during the $66 \mathrm{MY}$ of evolution. These rate estimates are among the lowest we have obtained to date ${ }^{14,15}$. Furthermore, $43.7 \%$ of the J. regia genes were collinear with genes on the grape pseudomolecules, which is remarkable considering that the two lineages diverged more than 100 MYA. This high gene collinearity among woody perennials contrasts with low gene collinearity in comparisons involving genomes of herbaceous plants, such as those of grasses (Table S16), and provide additional evidence for slow genome evolution in woody perennials ${ }^{17}$.

\section{Materials and methods \\ Trees}

DJUG 31.01 is a tree grown from J. microcarpa seeds collected near Sanderson TX USA by Loy Shreve as accession 83-131. Serr is the result of a cross between $J$. regia cv Payne of unknown seedling origin and PI 159568, a USDA plant introduction of $J$. regia from Paghman, Afghanistan. We generated clones of their hybrid MS1-56 by micropropagating the epicotyl excised from an immature hybrid embryo and transplanted rooted clones into pots in a greenhouse.

\section{Optical map construction}

We collected leaves from tissue culture-grown clones for MS1-56 and orchard trees for Serr and 31.01 and provided them to Amplicon Express (Pullman WA USA) for high-molecular-weight (HMW) ( $>500 \mathrm{~kb})$ DNA isolation. Based on the frequency of recognition sites in the genome sequence of $J$. regia cv Chandler ${ }^{11}$, we selected nicking endonucleases Nt.Bsp QI and Nb.BssSI (New England BioLabs, Ipswich MA USA) for DNA nicking. We treated HMW DNA of MS1-56 separately with both enzymes and that of Serr and 31.01 only with Nt.BspQI. We stained nicked molecules according to the IrysPrep Reagent Kit instructions [Bionano Genomics, San Diego CA USA, (henceforth BNG)] and imaged the stained DNA molecules with the Irys system (BNG). To obtain basic labelling and length information, we converted molecules $>20 \mathrm{~kb}$ into bnx files with AutoDetect (BNG) software. We then aligned molecules $>180 \mathrm{~kb}$ that passed the quality control against each other, clustered them, and assembled them into contigs using the BNG assembly pipeline $e^{53,54}$. We used the $p$-values $1 \times 10^{-8}, 1 \times 10^{-9}$, and $1 \times 10^{-15}$ as thresholds for pairwise assembly, extension/ refinement, and final contig refinement, respectively. We manually checked the resulting optical maps for chimeric contigs and resolved them when detected.

\section{Optical map length comparisons}

Optical maps of Serr and 31.01 consisted of diploid and haploid regions. We self-aligned optical maps to identify diploid regions, decided which of the two haplotypes in the phased regions were present on the MS1-56 optical map, and disregarded the alternative haplotype. The sum of the edited Serr and 31.01 optical map lengths was compared with the length of the MS1-56 optical map.

\section{Pacific biosciences and 10X genomics sequencing}

We isolated nuclear DNAs from MS1-56 as described earlier ${ }^{55}$ and prepared sequencing libraries using protocols from Pacific Biosciences (Menlo Park CA USA, 
henceforth). They were sequenced with the Single Molecule Real-Time (SMRT) technology at the Genome Center, University of California, Davis on the PacBio long-read sequencing instrument RSII using the P6 Polymerase Binding and C4 Chemistry Kits. Sequencing of the 58 PacBio SMRT cells of MS1-56 generated 57.2 Gb ( 54X coverage) of reads averaging $12,791 \mathrm{bp}$ with a maximum length of $82,702 \mathrm{bp}$. We assembled 2.05 million errorcorrected qualified reads with Falcon (v0.4.2) ${ }^{56}$ into 460 contigs (total length of $1,056,053,412 \mathrm{bp}$ ), which we polished with the genomic consensus tool Quiver (https:// github.com/PacificBiosciences/GenomicConsensus). We named this assembly MS1-56_v0.

To prepare 10X Genomics sequencing library, we isolated DNA from leaves of orchard-grown MS1-56. We prepared a sequencing library according to a protocol from 10X Genomics (Pleasanton CA USA). We sequenced the library with Illumina HiSeq4000 and assembled 680.53 million reads (89X genome equivalent, Table S1) with the 10X Genomics software Supernova $(\mathrm{v} 1.1 .3)^{57}$ using default parameters. The assembly produced 79,926 contigs with a total length of 1,005,111,912 bp and an N50 of 41,627 bp.

\section{Scaffolding}

We digested 460 MS1-56 sequence contigs in assembly MS1-56_v0 in silico with the Nt.BspQI and Nb.BssSI restriction endonucleases using Knickers (BNG) and aligned the contigs on the MS-156 Nt.Bsp QI and Nb. BssSI optical maps using RefAligner (BNG). We visually checked the alignments in IrysView (BNG). Of the 460 MS1-56 polished contigs, 239 ( $1047 \mathrm{Mb})$ were of sufficient length to be aligned. Five of the aligned contigs were chimeric and we disjoined them, increasing the total number of polished PacBio contigs to 465 (assembly MS1-56_v1, Table S2). The contig N50 =7,904,778 bp. We then aligned MS1-56_v1 contigs on optical maps and scaffolded them with the hybrid-scaffolding pipeline (BNG). Since two optical maps were generated for MS156, we performed a two-step hybrid-scaffolding resolution with the strictest 'conflict-free' setting, first for sequence contigs aligned on the Nt.Bsp QI optical map. We then aligned scaffolds on the Nb.BssSI optical map and scaffolded them further. We performed scaffolding also in the reverse order, using the $\mathrm{Nb}$.BssSI optical map for aligning sequence contigs, which was then followed by scaffolding on the Nt.BspQI optical map. We found no difference between the two approaches. BNG (https:// bionanogenomics.com/support/software-downloads/) provided us with software packages for scaffolding.

To stitch the scaffolds, we trained the filtering parameters of Stitch ${ }^{58}$ to be suitable for the MS1-56 genome and then performed Stitch in iterations until we could produce no additional scaffolds. After each round of
Stitch, we manually checked the 'potentially chimeric scaffolds' flagged by the program and resolved them if necessary. This sequential use of Stitch reduced the number of scaffolds from 465 to 264. The total length of this assembly (MS1-56_scf) was 1,066,408,726 bp, N50= $34,776,948 \mathrm{bp}$, and $0.97 \%$ Ns.

\section{Phasing MS1-56 scaffolds into parental genomes}

We aligned the 264 scaffolds of the MS1-56_scf assembly on the Serr and 31.01 optical maps using RefAligner (BNG). Of the 1,066,408,726 bp of the MS1-56 sequence, we allocated $536,956,484,537 \mathrm{bp}$ present in 19 scaffolds to Serr and $527,846,219 \mathrm{bp}$, present in 21 scaffolds, to 31.01 . This accounted for $99.85 \%$ of the assembly. The remaining 224 MS1-56 scaffolds had a total length of $1,606,023 \mathrm{bp}(0.15 \%$ of the assembly) and were too short to be aligned on optical maps. We allocated those that could be aligned on the sequence of Chandler ${ }^{11}$ (88 scaffolds) and the remainder (136) we allocated to 31.01 by default. Thus, we allocated all 264 scaffolds to one of the two parental genomes: 107 to the Serr genome (JrSerr_v0.1), totaling $538,152,081 \mathrm{bp}$, and 157 to the 31.01 genome (Jm31.01_v0.1) totaling 528,256,645 bp.

\section{Pseudomolecule construction, gap closing, and error corrections}

Eighteen Serr scaffolds equivalent to $532,266,604 \mathrm{bp}$ and representing $98.9 \%$ of the Serr assembly and 19 of the 31.01 scaffolds equivalent to $523,690,948 \mathrm{bp}$ and representing $99.1 \%$ of the 31.01 assembly shared SNP markers with the J. regia genetic and physical maps ${ }^{17}$ and a J. microcarpa genotyping-by-sequencing-produced map. We used these SNPs to ordered and orient scaffolds on the maps to construct pseudomolecules. The remaining 89 scaffolds $(5,885,477 \mathrm{bp})$ of Serr and 138 scaffolds $(4,565,697 \mathrm{bp})$ of 31.01 were short and could not be included into the pseudomolecules. There were two gaps of unknown size in the JrSerr_v0.1 pseudomolecules and three such gaps in the Jm31.01_v0.1 pseudomolecules, which we filled with $1,000 \mathrm{Ns}$. We estimated the lengths of the remaining 102 gaps in the JrSerr_v0.1 pseudomolecules and 106 in the Jm31.01_v0.1 pseudomolecules based on sequence alignments on the optical maps. The numbers and lengths of these gaps were reduced with unassigned scaffolds or sequence contigs assembled from 10X Genomics reads to 54 in both pseudomolecule sets with software FGAP v1.8.1 $1^{59}$.

To correct errors remaining in the pseudomolecule sequences, we mapped about $93.9 \mathrm{~Gb}$ of Illumina reads $(89 \times)$ onto the JrSerr_v0.1 and Jm31.01_v0.1 pseudomolecules using BWA v0.7.5 $5^{60}$. We removed the duplicate reads present among the Illumina reads generated by PCR with SAMtools $0.1 .19^{61}$. We performed single sample 
variant calling with SAMtools and BCFtools $0.1 .19^{62}$ and kept variants with read depth $>10$ and $<200$ and genotype quality $>30$ and discarded the rest. We also discarded heterozygous read stacks. We aligned Illumina reads on the pseudomolecule sequences and corrected base substitutions and indels in the pseudomolecules with VCFtools $^{63}$, which generated the final version of pseudomolecules and unanchored scaffolds JrSerr_v1.0 and Jm31.01_v1.0.

\section{TE annotation and SSR discovery}

We identified repeated sequences in the JrSerr_v1.0 and Jm31.01_v1.0 assemblies by a combination of homologbased and de novo approaches. We used the software RepeatModeler v1.0.10 (http://www.repeatmasker.org/ RepeatModeler) to obtain TE consensus sequences. We deployed MITE-Hunter ${ }^{64}$ to identify Miniature Inverted Repeat Transposable Elements (MITEs) and SINEFinder $^{65}$ to identify Short Interspersed Nuclear Elements (SINEs). We manually inspected the outputs to eliminate artifacts and combined the TEs which passed the quality control with the current release of Repbase v22.06 ${ }^{66}$ and the $J$. regia repetitive library to produce a custom TE library. We used the custom library to predict TEs with software RepeatMasker v4.0.7 (http://www.repeatmasker. org). We classified the TE sequences according to the system proposed by Wicker et al. ${ }^{67}$.

We used the MISA ${ }^{68}$ software to identify microsatellite motifs in the assemblies with the following minimum repeat numbers: ten for monomers, six for the di-, and five for the tri-, tetra-, penta-, and hexanucleotide motifs. We used a 1-Mb sliding window to calculate the SSR density on each chromosome.

\section{Gene annotation and transcription factor and $\mathrm{R}$ gene identification}

We used both homology-based and de novo methods to predict protein-coding genes in the TE masked JrSerr_v1.0 and Jm31.01_v1.0 assemblies. We extracted homologous protein sequences for eudicots from NCBI and used them as training sets in Augustus ${ }^{69}$. We used a J. regia gene set ${ }^{11}$ to create species-specific parameters for Augustus and used these parameters also in de novo gene prediction by $\mathrm{Maker}^{70}$. For homology-based gene annotation, we used the transcripts of $J$. regia ${ }^{11}$ to generate predicted homology-based gene models in Maker ${ }^{70}$. Finally, we merged the gene models from de novo prediction and homology-based prediction to produce a comprehensive and non-redundant gene set by using Maker ${ }^{70}$.

We used the Benchmarking Universal Single-Copy Orthologs (BUSCO) v3 tool ${ }^{71}$ to identify universal single copy orthologs (USCOs) in the assembly as a measure of completeness. BUSCO analysis was performed using the plant dataset (embryophyta_odb9), which excludes the
Physcomitrella patens and Selaginella moellendorffi and contained 1440 BUSCO groups.

We used DNA-binding domains in Pfam $31.0^{72}$ to identify transcription factors (TFs) among the proteincoding genes annotated in JrSerr_v1.0 and Jm31.01_v1.0 using BLAST. We classified the TFs according to the Plant Transcription Factor Databases (plantTFDB, http:// planttfdb.cbi.edu.cn/).

$\mathrm{R}$ genes were identified using the prediction pipeline RGAugury ${ }^{73}$. Amino acid sequences of the protein coding genes in the whole genomes and their coordinates on the genomes (gff3 files) were used as the input. An E value of $1 \times 10^{-5}$ was used for the protein BLAST search against four protein databases: pfam, smart, gene3d, and superfamily. Four types of RGAs were predicted, including nucleotide-binding sites encoding genes, RLK, receptorlike protein, and transmembrane coiled-coil protein through screening for the presence of major domains, such as NB-ARC, toll-interleukin receptor, leucine-rich repeats, coiled-coil domain, transmembrane domain, serine/threonine kinase domain, and LysM motif.

To determine if the distribution of the $\mathrm{R}$ genes along chromosomes mirrored that of other genes, the total number of genes along a chromosome arm were divided into 20 windows, each containing the same number of genes. The proportion of $\mathrm{R}$ genes in each window was computed and averaged across the 32 chromosome arms. Rank correlation coefficients $(r)$ for the window location on the telomere-to-centromere axis and the mean proportion of $\mathrm{R}$ genes in the window were computed for both genome assemblies.

\section{Dot-plots and circular plots}

To construct synteny dot-plots, we downloaded protein sequences for Vitis vinifera (Genoscope ${ }^{18}$ and Amborella trichopoda v1.0 ${ }^{19}$ from Phytozome v12.0 and used them in BLASTP searches as targets while genes from JrSerr_v1.0 were used as queries. We also aligned JrSerr_v1.0 and Jm31.01_v1.0, in which we used the former as a query source and the latter as a target in BLASTP with an $\mathrm{E}$ value of $1 \times 10^{-5}$. We analyzed all alignments with $\mathrm{MCScanX} \mathrm{X}^{74}$ with default parameters. We also use MCScanX with default parameters to find collinear blocks containing at least five collinear gene pairs. We visualized syntenic blocks in Circos $0.69^{75}$ and constructed the dotplots with dot_plotter in MCScanX ${ }^{74}$.

To identify WGD within J. regia, we searched homology by BLASTP using HC genes annotated on the JrSerr_v1.0 pseudomolecules both as queries and targets (self-search). We performed the same analysis with $\mathrm{HC}$ genes annotated on the Jm31.01_v1.0 pseudomolecules. We used a filter threshold of $1 \times 10^{-5}$ to identify homologous proteins. The longest protein sequences were analyzed with MCScanX with default parameters. We considered at 
least five genes in an ascending or descending order as a syntenic block. We constructed the syntenic block maps with Circos 0.69 software $^{75}$ and dot-plots with dot_plotter in MCScanX ${ }^{74}$.

\section{Molecular clock rate}

We used the JrSerr_v1.0, Jm31.01_v1.0, $V$. vinifera, and $A$. trichopoda gene sequences to calculate the synonymous $(K s)$ substitution rates. We identified orthologous genes among these four species using Proteinortho $5^{76}$. We identified orthologous inter-genome gene pairs and paralogous intra-genome gene pairs by bi-directional BLAST with the default parameter. We aligned aminoacid sequences of each pair of genes using ClustalW ${ }^{77}$ and transformed amino-acid alignments into nucleotide sequence alignments according to coding sequences (CDS). We then calculated the $K a$ and $K s$ of each pair of genes using Kimura 2 parameter model in KaKs_Calculator $2.0^{78}$. We computed genome divergence times and dated the Juglandoid WGD or $\gamma$ WGT according to $r=$ $K s / 2 t$, where $r$ is the rate of nucleotide substitution per synonymous site per year, $K s$ is the number of substitutions per synonymous site, and $t$ is time of species divergence in years. We used the same approach to compute $r$ from $\mathrm{Ka}$. Using the time estimate and the average $K a$ and $K s$ between paralogues on the homoeologous chromosomes in the Serr and 31.01 genomes, we computed clock rates.

\section{Gene collinearity and structural chromosome analyses}

The methodology used for gene collinearity analysis has been described earlier ${ }^{14}$, and we will provide only essential information. We used the amino-acid sequences of 31,286 HC genes annotated in the JrSerr_v1.0 pseudomolecules as a query and the amino acid sequences of grape $\mathrm{HC}$ genes as targets in BLASTP homology searches. We used a default BLASTP parameter setting. We also performed BLSTP search using JrSerr_v1.0 as a query against itself (target). We recorded the top three alignments. In the BLASTP searches using JrSerr_v1.0 as a query against Jm31.01_v1.0 as a target, we recorded only the top hit.

We analyzed collinearity as follows. We considered three or more genes to be collinear if the starting nucleotides of the top hits followed an ascending or descending order and distances between them were $<0.5 \mathrm{Mb}$, allowing non-collinear genes to interrupt a sequence of collinear genes. If a gene was homologous to tandem duplicated genes on a target pseudomolecule, we recorded only one of the duplicated genes as collinear, provided that it was in a collinear position on the pseudomolecule.

We constructed a spreadsheet of datasets showing collinearity of each of the 31,286 genes on the JrSerr_v1.0 pseudomolecules with those on the JrSerr_v1.0 pseudomolecules (self), with those on the Jm31.01_v1.0 pseudomolecules, and the grape pseudomolecules. We colored spreadsheet cells containing collinear genes whereas those that were not collinear we left colorless. We indicated changes in gene order due to inversions or translocations by changes in cell color. We recorded for each rearrangement the register of the starting and ending gene and rearrangement type. We coded the rearrangements as follows. $\mathrm{A}=$ inversion of 2 genes, $\mathrm{B}=$ inversion of 3 genes, $\mathrm{C}=$ inversion of $>3$ genes, $\mathrm{D}=$ translocation of 2 genes within a chromosome, $\mathrm{E}=$ translocation of 3 genes within a chromosome, $\mathrm{F}=$ translocation $>3$ genes within a chromosome, $\mathrm{iT}=$ interstitial translocation between chromosomes, $\mathrm{T}=$ terminal translocation, Dup $=$ duplication of a segment, and Del = deletion of a segment. To quantify collinearity, we counted genes of JrSerr_v1.0 collinear with a specific genome and expressed them as a percentage of all $\mathrm{HC}$ genes present on the JrSerr_v1.0 pseudomolecules.

We compared the order of collinear genes along pseudomolecules within JrSerr_v1.0, between JrSerr_v1.0 and Jm31.01_v1.0, and between JrSerr_v1.0 and the grape pseudomolecules and determined the most parsimonious origin of a rearrangement, provided that information on the rearrangement was available in all genomes and subgenomes. We coded the branch assignments as follows: J. microcarpa (M), J. regia (R), and the branch preceding the divergence of the two (RM). We used only major rearrangements $(\mathrm{C}, \mathrm{F}$, and $\mathrm{iT})$ in computing rates. We computed the rearrangement rate acquisition by a branch using time estimates in Table S13.

We used the following approach to quantify gene collinearity between the JrSerr_v1.0 pseudomolecules and the grape pseudomolecules. We recorded only the top hits in an effort to identify the orthologous pseudomolecule among the three homoeologous pseudomolecules in the grape genome. Since this approach still produced multiple alignments for every JrSerr_v1.0 pseudomolecule in the grape genome, we selected the alignment that contained the largest number of collinear genes and eliminated the remaining alignments present. We color coded the collinear genes on the orthologous alignments and counted them.

\section{Fractionation of the Juglandoid WGD}

To investigate the distribution of the differences in gene numbers along the dominant and subdominant chromosomes, we subdivided each homoeologous chromosome pair into $N$ intervals between $N+1$ collinear genes shared by the homoeologous chromosome pairs in the Serr genome. We then counted the number of unique genes (henceforth singletons) present in an interval and plotted the numbers for each homoeologue. We tested the significance of the difference in singleton numbers between 
the dominant and subdominant chromosomes with the paired $t$-test using the numbers in individual intervals in the dominant and subdominant chromosomes as variables.

To express the number of singletons per $\mathrm{Mb}$ rather than per interval between collinear genes, we subdivided the dominant chromosome into $2-\mathrm{Mb}$ intervals and counted and plotted the number of singleton genes in the $2-\mathrm{Mb}$ interval.

\section{Gene expression between dominant and subdominant chromosomes of $J$. regia}

We downloaded the expression data for 22 RNAseq libraries ${ }^{11}$ and measured dominance in gene expression between pairs of collinear orthologues located on the eight homoeologous chromosome pairs of J. regia. We quantified gene expression level using FPKM values in Cufflinks ${ }^{79}$. We considered collinear orthologous pairs with greater than two-fold difference ${ }^{80}$ as differentially expressed. We termed the gene that showed higher expression in differentially expressed gene pairs as the dominant orthologue whereas the gene with lower expression as the subordinate collinear orthologue. We counted the number of dominant genes in each chromosome and performed paired $t$-test to compare the difference in the number of dominant genes between dominant and subdominant subgenomes.

\section{Phylogeny reconstruction}

We downloaded the following genome assemblies for phylogenetic analysis and the construction of divergence chronology: Vitis vinifera ${ }^{18}$, Prunus persica ${ }^{81}$, Amborella trichopoda ${ }^{19}$, Populus trichocarpa ${ }^{35}$, Theobroma cacao $^{82}$, Malus domestica ${ }^{83}$, and J. regia Chandler, J. sigillata, J. nigra, J. microcarpa acc. 29.01, J. hindsii, J. cathayensis, Pterocarya stenoptera ${ }^{5}$. With Proteinortho ${ }^{76}$ using default parameters we identified 1155 single-copy, orthologous genes shared by the J. regia (JrSerr_v1.0), J. microcarpa (Jm3101_v1.0), Amborella trichopoda, Vitis vinifera, Malus domestica, Theobroma cacao, Prunus persica, and Populus trichocarpa genomes. We aligned amino-acid sequences of the 1,155 genes in the eight genomes with MAFFT $^{84}$, removed regions with gaps, and concatenated the remaining sequences. We used the phylogenetic software IQ-TREE ${ }^{85}$ employing the Maximum-likelihood method to infer the phylogeny of the eight genomes and performed 1000 bootstrap replications to produce a consensus tree. We constructed the final phylogenetic tree with Figtree ${ }^{86}$.

Because neither pseudomolecules nor gene annotation was reported for J. regia Chandler, J. sigillata, J. nigra, J. microcarpa acc. 29.01, J. hindsii, J. cathayensis, and Pterocarya stenoptera ${ }^{5}$, we aligned the CDS sequences of the 1,155 Serr and 31.01 genes to these seven genomic sequences to obtain corresponding CDS sequences. We then constructed a phylogenetic tree for the fifteen genomes as described above with the following changes. We converted the CDS sequences to amino-acid sequences. After removing partial alignments, all 15 genomes shared 809 single-copy, orthologous genes. We aligned the gene sequences with MAFFT $^{84}$, removed regions with gaps, concatenated the remaining sequences, and constructed a tree.

\section{Divergence time estimation}

Based on fossil record, the diversification of Juglandaceae was estimated to have been initiated 80 MYA (71-96 MYA $95 \% \mathrm{CI})^{24}$. Since this estimate includes speciation of diploid Rhoiptelea, the WGD must have occurred after this date. Based on the chronogram in ${ }^{24}$ and fossil dating of the Engelhardioideae and Juglandoideae divergence to upper Cretaceous ${ }^{21,87}$ we arbitrarily chose 66 MYA for the origin of the Juglandoid WGD (reviewed $\mathrm{in}^{88}$ ). Based on the non-synonymous $(\mathrm{Ka})$ and synonymous $(K s)$ divergence values computed for 1,429 collinear genes on the Serr homoeologous chromosome with KaKs Calculator $2.0^{78}$, we calibrated the molecular clock. We used $\mathrm{MACSE}^{89}$ to perform multiple sequence alignment for each gene using the method of model averaging. The divergence $(k)$ of pairs of orthologous genes was converted into time of divergence $(t)$ in million years using formula: $t=k /(2 r) / 10^{6}$.

\section{Acknowledgements}

This material is based upon work supported by USDA/NIFA/SCRI under grant number 59-5306-2-333 and by the California Walnut Board.

\section{Author details}

'Department of Plant Sciences, University of California, Davis, CA 95616, USA. ${ }^{2}$ Ottawa Research and Development Centre, Agriculture and Agri-Food Canada, Ottawa, ON K1A 0C6, Canada. ${ }^{3}$ Genome Center, University of California, Davis, CA 95616, USA. ${ }^{4}$ Crops Pathology and Genetics Research Unit, USDA-ARS, Davis, CA 95616, USA. ${ }^{5}$ National Clonal Germplasm Repository, USDA-ARS, Davis, CA 95616, USA

\section{Author contributions}

M.C.L., J.D., D.K., and M.A. conceived the study. T.Z., L.W., C.A.L., L.C., K.R.D., and M.C.L. planned, organized, and conducted the sequencing steps. J.L. performed sequence assembly. T.Z., J.C.R., K.R.D., and M.C.L. planned, organized, and conducted construction of Bionano optical maps. T.Z., L.W., and M.-C.L. conducted genome assembly. L.W. performed TE annotation. F.M.Y., L.W., S.C., B.B., C.Z.J., and A.M.D. contributed to gene annotations. L.W., F.M.Y., J. D., M.-C.L., P.J.B., and P.E.M contributed to comparative analysis and phylogeny analysis. J.D., M.C.L., T.Z., and L.W. drafted the manuscript with everyone's inputs on relevant sections. All authors approved the final manuscript.

\section{Data availability}

The genome assemblies and gene annotations of $J$. regia Serr and $J$. microcarpa acc. 31.01, and all sequencing data have been deposited under NCBI BioProject PRJNA413991. The assemblies, TE and gene annotations of J. regia Serr and J. microcarpa acc. 31.01 are also accessible via the JBroswer at http://aegilops.wheat.ucdavis.edu/jbrowse/index.html?data $=\mathrm{JrSerr \& loc}$ and http://aegilops.wheat.ucdavis.edu/jbrowse/index.html?data $=$ Jm31.01\&loc. 


\section{Conflict of interest}

The authors declare that they have no conflict of interest.

Supplementary Information accompanies this paper at (https://doi.org/ 10.1038/s41438-019-0139-1).

Received: 4 February 2019 Revised: 9 March 2019 Accepted: 10 March 2019 Published online: 25 March 2019

\section{References}

1. Zhang, F. et al. Haplotype phasing of whole human genomes using beadbased barcode partitioning in a single tube. Nat. Biotechnol. 35, 852-857 (2017).

2. Schnable, P. S. et al. The B73 maize genome: complexity, diversity, and dynamics. Science 326, 1112-1115 (2009).

3. $\mathrm{Xu}, \mathrm{Q}$. et al. The draft genome of sweet orange (Citrus sinensis). Nat. Genet. 45 59-66 (2013).

4. Neale, D. B., Martínez-García, P. J., De La Torre, A. R., Montanari, S. \& Wei, X. X. Tree genome sequencing: Novel insights into plant biology. Ann. Rev. Plant Biol. 68, 457-483 (2017).

5. Stevens, K. A. et al. Genomic variation among and within six Juglans species. G3: Genes/Genomes|Genetics (2018). https://doi.org/10.1534/g3.118.200030

6. Pan, A., Sun, Q., Manson, J. E., Willett, W. C. \& Hu, F. B. Walnut consumption is associated with lower risk of type 2 diabetes in women. J. Nutr. 143, 512-518 (2013).

7. Kris-Etherton, P. M. Walnuts decrease risk of cardiovascular disease: a summary of efficacy and biologic mechanisms. J. Nutr. 144, 547s-554s (2014).

8. Poulose, S. M., Miller, M. G. \& Shukitt-Hale, B. Role of walnuts in maintaining brain health with age. J. Nutr. 144, 561s-566s (2014).

9. Browne, G. T. et al. Resistance to Species of Phytophthora identified among clones of Juglans microcarpa $x$ J. regia. Hortsci 50, 1136-1142 (2015).

10. Kluepfel, D. A. et al. Evaluation of wild walnut Juglans spp. for resistance to crown gall disease. Phytopathology 101, S92-S92 (2011).

11. Martinez-Garcia, P. J. et al. The walnut (Juglans regia) genome sequence reveals diversity in genes coding for the biosynthesis of non-structural polyphenols. Plant J. 87, 507-532 (2016).

12. Bai, W. N. et al. Demographically idiosyncratic responses to climate change and rapid Pleistocene diversification of the walnut genus Juglans (Juglandaceae) revealed by whole-genome sequences. New Phytol. 217, 1726-1736 (2018).

13. Zimin, A. V. et al. Hybrid assembly of the large and highly repetitive genome of Aegilops tauschii, a progenitor of bread wheat, with the MaSuRCA megareads algorithm. Genome Res. 27, 787-792 (2017).

14. Luo, M. C. et al. Genome sequence of the progenitor of the wheat D genome Aegilops tauschii. Nature 551, 498-502 (2017).

15. Dvorak, J. et al. Structural variation and rates of genome evolution in the grass family seen through comparison of sequences of genomes greatly differing in size. Plant J. 95, 487-503 (2018).

16. Zhu, T. et al. Analysis of Brachypodium genomes with genome-wide optical maps. Genome 61, 559-561 (2018).

17. Luo, M. C. et al. Synteny analysis in Rosids with a walnut physical map reveals slow genome evolution in long-lived woody perennials. BMC Genom. 16, 707 (2015).

18. Jaillon, $\mathrm{O}$. et al. The grapevine genome sequence suggests ancestral hexaploidization in major angiosperm phyla. Nature 449, 463-467 (2007).

19. Albert, V. A. et al. The Amborella genome and the evolution of flowering plants. Science 342, 1467 (2013).

20. Manos, P. S. \& Stone, D. E. Evolution, phylogeny, and systematics of the Juglandaceae. Ann. Mo. Bot. Gard. 88, 231-269 (2001).

21. Manchester, S. R. The fossil history of Juglandaceae. Monographs In Systematic Botany from the Missouri Botanical Garden. 21, 1-137 (1987).

22. Manchester, S. R. Early history of the Juglandaceae. Plant Syst. Evol. 162, 231-250 (1989).

23. Zhang, J. B. et al. Integrated fossil and molecular data reveal the biogeographic diversification of the Eastern Asian-Eastern North American Disjunct Hickory Genus (Carya Nutt.). PLOS. ONE. https://doi.org/10.1371/journal. pone.0070449 (2013).
24. Xiang, X. G. et al. Large-scale phylogenetic analyses reveal fagalean diversification promoted by the interplay of diaspores and environments in the Paleogene. Perspect. Plant Ecol. 16, 101-110 (2014).

25. Dong, W. P. et al. Phylogenetic resolution in juglans based on complete chloroplast genomes and nuclear DNA sequences. Front. Plant Sci. 8, 1148 (2017).

26. Clarke, J. T., Warnock, R. C. M. \& Donoghue, P. C. J. Establishing a time-scale for plant evolution. New Phytol. 192, 266-301 (2011).

27. Jiao, Y. N. et al. A genome triplication associated with early diversification of the core eudicots. Genome Biol. 13, R3 (2012).

28. Zeng, L. P. et al. Resolution of deep eudicot phylogeny and their tempora diversification using nuclear genes from transcriptomic and genomic datasets. New Phytol. 214, 1338-1354 (2017).

29. Presting, G. G., Malysheva, L., Fuchs, J. \& Schubert, I. Z. A TY3/GYPSY retrotransposon-like sequence localizes to the centromeric regions of cereal chromosomes. Plant J. 16, 721-728 (1998).

30. Sharma, A. \& Presting, G. G. Evolution of centromeric retrotransposons in grasses. Genome Biol. Evol. 6, 1335-1352 (2014).

31. Luo, S. et al. The cotton centromere contains a Ty3-gypsy-like LTR retroelement. PLOS. ONE 7, 35261 (2012).

32. Tek, A. L., Kashihara, K., Murata, M. \& Nagaki, K. Functional centromeres in soybean include two distinct tandem repeats and a retrotransposon. Chrom. Res. 18, 337-347 (2010).

33. Weber, B. \& Schmidt, T. Nested Ty3-gypsy retrotransposons of a single Beta procumbens centromere contain a putative chromodomain. Chrom. Res. 17, 379-396 (2009).

34. Lim, K. B. et al. Characterization of the centromere and peri-centromere retrotransposons in Brassica rapa and their distribution in related Brassica species. Plant J. 49, 173-183 (2007).

35. Tuskan, G. A. et al. The genome of black cottonwood, Populus trichocarpa (Torr. \& Gray). Science 313, 1596-1604 (2006).

36. Velasco, E. A. A high quality draft consensus sequence of the genome of a heterozygous grapevine variety. PLOS. ONE 2, 326 (2007).

37. Velasco, R. et al. The genome of the domesticated apple (Malus $x$ domestica Borkh.). Nat. Genet. 42, 833-839 (2010).

38. Minio, A., Lin, J., Gaut, B. S. \& Cantu, D. How Single molecule real-time sequencing and haplotype phasing have enabled reference-grade diploid genome assembly of wine grapes. Front. Plant Sci. 8, 826 (2017).

39. Schmutz, J. et al. Quality assessment of the human genome sequency. Nature 429, 365-368 (2004)

40. Ho, K. M. \& Kasha, K. J. Genetic control of chromosome elimination during haploid formation in barley. Genetics 81, 263-275 (1975).

41. Song, K. M., Lu, P., Tang, K. L. \& Osborn, T. C. Rapid genome change in synthetic polyploids of Brassica and its implications for polyploid evolution. Proc. Natl Acad. Sci. USA 92, 7719-7723 (1995).

42. Liu, B. et al. Rapid genomic changes in newly synthesized amphiploids of Triticum and Aegilops. I. changes in low-copy noncoding DNA sequences. Genome 41, 272-277 (1998).

43. Shaked, H., Kashkush, K., Ozkan, H., Feldman, M. \& Levy, A. A. Sequence elimination and cytosine methylation are rapid and reproducible responses of the genome to wide hybridization and allopolyploidy. Plant Cell $\mathbf{1 3}$ 1750-1759 (2001)

44. Osborn, T. C. et al. Understanding mechanisms of novel gene expression in polyploids. Trends Genet. 19, 141-147 (2003)

45. Oleszczuk, S. \& Lukaszewski, A. J. The origin of unusual chromosome constitutions among newly formed allopolyploids. Am. J. Bot. 101, 318-326 (2014).

46. Langham, R. J. et al. Genomic duplication, fractionation and the origin of regulatory novelty. Genetics 166, 935-945 (2004).

47. Dvorak, J., Yang, Z.-L., You, F. M. \& Luo, M. C. Deletion polymorphism in wheat chromosome regions with contrasting recombination rates. Genetics $\mathbf{1 6 8}$ 1665-1675 (2004).

48. Cheng, F. et al. Biased gene fractionation and dominant gene expression among the subgenomes of Brassica rapa. PLOS. ONE 7, 36442 (2012).

49. Renny-Byfield, S., Gong, L., Gallagher, J. P. \& Wendel, J. F. Persistence of subgenomes in paleopolyploid cotton after 60 My of evolution. Mol. Biol. Evol. 32 , 1063-1071 (2015)

50. Schnable, J. C., Springer, N. M. \& Freeling, M. Differentiation of the maize subgenomes by genome dominance and both ancient and ongoing gene loss. Proc. Natl Acad. Sci. USA 108, 4069-4074 (2011). 
51. Renny-Byfield, S., Rodgers-Melnick, E. \& Ross-lbarra, J. Gene fractionation and function in the ancient subgenomes of maize. Mol. Biol. Evol. 34, 1825-1832 (2017).

52. Woodhouse, M. R. et al. Origin, inheritance, and gene regulatory consequences of genome dominance in polyploids (vol 111, pg 5283, 2014). Proc. Natl Acad. Sci. USA 111, 6527-6527 (2014)

53. Lam, E. T. et al. Genome mapping on nanochannel arrays for structural variation analysis and sequence assembly. Nat. Biotechnol. 30, 771-776 (2012).

54. Cao, H. Z. et al. Rapid detection of structural variation in a human genome using nanochannel-based genome mapping technology. Gigascience 3, 34 (2014).

55. Dvorak, J., McGuire, P. E. \& Cassidy, B. Apparent sources of the A genomes of wheats inferred from the polymorphism in abundance and restriction fragment length of repeated nucleotide sequences. Genome 30, 680-689 (1988).

56. Chin, C. S. et al. Phased diploid genome assembly with single-molecule realtime sequencing. Nat. Methods 13, 1050-1054 (2016).

57. Weisenfeld, N. I., Kumar, V., Shah, P., Church, D. M. \& Jaffe, D. B. Direct determination of diploid genome sequences. Genome Res. 27, 757-767 (2017).

58. Shelton, J. M. et al. Tools and pipelines for BioNano data: molecule assembly pipeline and FASTA super scaffolding tool. BMC Genom. 16, 734 (2015).

59. Piro, V. C. et al. FGAP: an automated gap closing tool. BMC Res. Notes 7, 371 (2014).

60. Li, H. \& Durbin, R. Fast and accurate long-read alignment with BurrowsWheeler transform. Bioinformatics 26, 589-595 (2010).

61. Li, H. et al. The Sequence Alignment/Map format and SAMtools. Bioinformatics 25, 2078-2079 (2009).

62. Li, H. A statistical framework for SNP calling, mutation discovery, association mapping and population genetical parameter estimation from sequencing data. Bioinformatics 27, 2987-2993 (2011).

63. Danecek, P. et al. The variant call format and VCFtools. Bioinformatics 27, 2156-2158 (2011)

64. Han, Y. J. \& Wessler, S. R. MITE-Hunter: a program for discovering miniature inverted-repeat transposable elements from genomic sequences. Nucleic Acids Res. 38, 199 (2010).

65. Wenke, T. et al. Targeted identification of short interspersed nuclear element families shows their widespread existence and extreme heterogeneity in plant genomes. Plant Cell 23, 3117-3128 (2011)

66. Bao, W., Kojima, K. K. \& Kohany, O. Repbase update, a database of repetitive elements in eukaryotic genomes. Mob. DNA 6, 11 (2015)

67. Wicker, T. et al. A unified classification system for eukaryotic transposable elements. Nat. Rev. Genet. 8, 973-982 (2007).

68. Thiel, T., Michalek, W., Varshney, R. K. \& Graner, A. Exploiting EST databases for the development and characterization of gene-derived SSR-markers in barley (Hordeum vulgare L.). Theor. Appl. Genet. 106, 411-422 (2003).

69. Stanke, M. et al. AUGUSTUS: ab initio prediction of alternative transcripts. Nucleic Acids Res. 34, W435-W439 (2006).

70. Cantarel, B. L. et al. MAKER: An easy-to-use annotation pipeline designed for emerging model organism genomes. Genome Res. 18, 188-196 (2008).
71. Simão, F. A. et al. BUSCO: assessing genome assembly and annotation completeness with single-copy orthologs. Bioinformatics 31, 3210-3212 (2016).

72. Finn, R. D. et al. The Pfam protein families database: towards a more sustainable future. Nucleic Acids Res. 44, D279-D285 (2016).

73. Li, P. C. et al. RGAugury: a pipeline for genome-wide prediction of resistance gene analogs (RGAs) in plants. BMC Genom. 17, 852 (2016).

74. Wang, Y. P. et al. MCScanX: a toolkit for detection and evolutionary analysis of gene synteny and collinearity. Nucleic Acids Res. 40, e49 (2012).

75. Krzywinski, M. et al. Circos: An information aesthetic for comparative genomics. Genome Res. 19, 1639-1645 (2009).

76. Lechner, M. et al. Proteinortho: detection of (co-)orthologs in large-scale analysis. BMC Bioinforma. 12, 124 (2011)

77. Thompson, J. D., Higgins, D. G. \& Gibson, T. J. Improved sensitivity of profile searches through the use of sequence weights and gap excision. Comput. Appl. Biosci. 10, 19-29 (1994)

78. Wang, D., Zhang, Y., Zhang, Z., Zhu, J. \& Yu, J. KaKs_Calculator 2.0: a toolkit incorporating gamma-series methods and sliding window strategies. Genom., Proteom., Bioinforma. 8, 77-80 (2010).

79. Trapnell, C. et al. Transcript assembly and quantification by RNA-Seq reveals unannotated transcripts and isoform switching during cell differentiation. Nat. Biotechnol. 28, 511-U174 (2010).

80. Zhang, T. Z. et al. Sequencing of allotetraploid cotton (Gossypium hirsutum L. acc. TM-1) provides a resource for fiber improvement. Nat. Biotechnol. 33 531-U252 (2015).

81. International Peach Genome, I. et al. The high-quality draft genome of peach (Prunus persica) identifies unique patterns of genetic diversity, domestication and genome evolution. Nat. Genet. 45, 487-494 (2013)

82. Argout, X. et al. The genome of Theobroma cacao. Nat. Genet. 43, 101-108 (2011).

83. Daccord, N. et al. High-quality de novo assembly of the apple genome and methylome dynamics of early fruit development. Nat. Genet. 49, 1099-1106 (2017).

84. Katoh, K., Misawa, K., Kuma, K. \& Miyata, T. MAFFT: A novel method for rapid multiple sequence alignment based on fast Fourier transform. Nucleic Acids Res. 30, 3059-3066 (2002).

85. Nguyen, L. T., Schmidt, H. A., von Haeseler, A. \& Minh, B. Q. IQ-TREE: A fast and effective stochastic algorithm for estimating maximum-likelihood phylogenies. Mol. Biol. Evol. 32, 268-274 (2015).

86. Rambaut, A. FigTree v1.4.3. http://tree.bio.ed.ac.uk (2009).

87. Manos, P. S. et al. Phylogeny of extant and fossil Juglandaceae inferred from the integration of molecular and morphological data sets. Syst. Biol. 56 412-430 (2007).

88. Chen, F. et al. Genomics: cracking the mysteries of walnuts. J. Genet. 98, (2019) in press.

89. Ranwez, V., Harispe, S., Delsuc, F. \& Douzery, E. J. MACSE: multiple alignment of coding sequences accounting for frameshifts and stop codons. PLOS. ONE 6, 22594 (2011). 